\title{
MENTES INDIVIDUALES E INTELECTO AGENTE UNIVERSAL EN LOS ESCRITOS LEIBNIZIANOS DE 1675-1676
}

\author{
Federico Raffo Quintana \\ IESCT-UNQ / CONICET
}

\begin{abstract}
RESUMEN: Algunos pasajes escritos por Leibniz en 1675/1676 parecerían sugerir que el filósofo de Leipzig mantuvo una concepción de tipo averroísta según la cual hay un único intelecto agente universal común a todas las mentes individuales. Al mismo tiempo, esta interpretación se complementa con la idea sostenida por algunos exégetas de que Leibniz, inspirándose en Spinoza, habría defendido que hay una única sustancia de la que las mentes singulares son modos. En este trabajo buscaremos mostrar que Leibniz no defendió un intelecto universal común a todas las mentes, a pesar de que admitió la existencia de una inteligencia primera. En el camino que nos conducirá hacia esto, justificaremos también que el filósofo de Leipzig mantuvo que existe una pluralidad de 'entes' tipo mentes.
\end{abstract}

PALABRAS CLAVE: Leibniz; mentes; intelecto agente; omnisciencia; averroísmo.

\begin{abstract}
Some passages written by Leibniz in 1675/1676 seem to suggest that the philosopher from Leipzig held an Averroistic conception, according to which there is a unique universal agent intellect common to all individual minds. At the same time, this interpretation is complementary with the idea (held by some commentators) that Leibniz, inspired by Spinoza, would have argued that there is a unique substance of which the individual minds are modes. In this paper we argue that Leibniz did not maintain a universal, common to all minds intellect, even though he admitted the existence of a primary intelligence. In showing this, we will also argue that the philosopher from Leipzig held that there is a plurality of 'beings' like minds.
\end{abstract}

KEYWORDS: Leibniz; minds; agent intellect; omniscience; Averroism.

\section{Introducción}

Desde hace algunos años se ha puesto de relieve la importancia del pensamiento de Leibniz en el tiempo de su estadía en París (1672-1676). Este período, que es conocido especialmente por los importantísimos estudios de Leibniz en el campo de la matemática (que comenzaron con sus exámenes sobre series infinitas y continuaron con algunos desarrollos que lo condujeron al 
cálculo infinitesimal), ${ }^{1}$ incluye también un grupo de escritos en los que los temas abordados pertenecen al dominio de la metafísica. Las traducciones de Parkinson de los escritos metafísicos de Leibniz de 1675-1676, ${ }^{2}$ agrupados en la edición canónica bajo el título de De summa rerum (de aquí en más, DSR), han sido un importante impulso para este tipo de exámenes. Ahora bien, el pensamiento metafísico de Leibniz del período de DSR es poco sistemático y muchas veces oscuro. Esto explica parcialmente la multiplicidad de interpretaciones que se han dado. Otra importante razón de la variedad de interpretaciones está relacionada con la posible influencia que Spinoza habría ejercido en Leibniz. Pese a que algunos especialistas, como Parkinson, hayan defendido que "no hay ninguna insinuación de (digamos) un criptoSpinozismo" en los textos de $1675-1676,{ }^{3}$ en general los estudiosos del pensamiento leibniziano de este período, como por ejemplo M. Kulstad o M. Laerke, arguyen que Spinoza podría haber influido en Leibniz, primero indirectamente, por medio de W. E. von Tschirnhaus (en este sentido, Laerke se refiere a un sistema 'cuasi-spinozista' en DSR), a quien Leibniz conoció en París, y luego directamente, tras el encuentro que mantuvieron ambos filósofos a finales de $1676 .{ }^{4} \mathrm{Al}$ menos parcialmente, esta posible influencia se justificaría

\footnotetext{
1 Para la labor matemática de Leibniz en sus años en París puede verse Hoffman 1974. Algunos escritos matemáticos de Leibniz de este período son, por ejemplo, Accessio ad arithmeticam infinitorum de 1672 (AA II 1 342-356), así como también De quadratura arithmetica circuli ellipseos et hyperbolae cujus coollarium est trigonometria sine tabulis de 1675/1676 (LEIBNIZ 2004).

2 Leibniz (1992).

${ }^{3}$ Leibniz (1992, xxi); similarmente, Mercer (2004), capítulo 10.

${ }^{4}$ Laerke (2008, pp.439-556); véase también Kulstad (2014).
} 


\section{Dossiê Leibniz, Dissertatio - Volume Suplementar 03 | UFPel [2016]}

en la presencia de un monismo de la sustancia en el pensamiento de Leibniz.

Esto puede observarse, por ejemplo, en este importante pasaje:

En síntesis, así como en el espacio hay algo divino, la inmensidad misma de Dios, del mismo modo en la mente hay algo divino, lo que Aristóteles llamaba intelecto agente; y esto es lo mismo que la omnisciencia de Dios. Del mismo modo que aquello que es divino y eterno en el espacio es lo mismo que la inmensidad de Dios, también aquello que es divino y eterno en el cuerpo o ente móvil es lo mismo que la omnipotencia de Dios, y también aquello que es divino en el tiempo es lo mismo que la eternidad. ${ }^{5}$

Este pasaje ha generado una discusión sobre si Leibniz ha mantenido o no una concepción de tipo averroísta, esto es, si ha entendido que hay un único intelecto agente universal común a todas las mentes. En efecto, Averroes (1126-1198) entendió que, como los inteligibles son únicos y eternos, el intelecto agente, esto es, el que los actualiza en el intelecto material, debe también serlo. En consecuencia, el intelecto agente es único y universal. ${ }^{6}$ De acuerdo con C. Wilson y E. De Tommaso, la indicación de Leibniz de que el intelecto agente es lo divino en las mentes es una expresión con implicancias que la hacen directamente afín con una concepción panteísta. ${ }^{7}$ En este sentido, la interpretación averroísta de Leibniz ha apoyado la tesis de que en el período de DSR habría defendido un monismo sustancial. Similarmente, M. Laerke, quien

\footnotetext{
${ }^{5}$ Notizen zur Wissenchaft und Metaphysik, AA VI 3, 391-392. "(...) prorsus ut in spatio est aliquid divinum, ipsa immensitas Dei, ita in mente est divinum quiddam, quod Aristoteles vocabat intellectum agentem, et hoc idem est cum omniscientia Dei; quemadmodum id quod in spatio divinum atque aeternum est, idem est cum Dei immensitate et id quod in corpore sive ente mobili divinum atque aeternum, idem est cum Dei omnipotentia; et id quod in tempore est divinum idem est cum aeternitate". Todas las traducciones de los escritos de Leibniz son nuestras. Véase también De origine rerum ex formis, AA VI 3 519-520.

${ }^{6}$ Averroes, Tafsir (2003), pp.36-37.

7 Wilson (1999), pp.224-228; De Tommaso (2014), p.102.
} 
argumenta a favor del 'unisustancialismo' en $D S R,{ }^{8}$ subraya que en sus escritos de juventud (especialmente en De transubstantiatione de 1668), Leibniz se mostró benévolo frente al averroísmo. ${ }^{9}$

Ahora bien, esta interpretación del texto de Leibniz ha supuesto un desafío para otra interpretación, como la de A. Blank, para quien en los escritos metafísicos de Leibniz de 1675-1676 se encuentra un pluralismo de sustancias activas tipo mentes que es compatible con el monismo de la sustancia divina. ${ }^{10}$ En efecto, Blank ha detectado y abordado el siguiente problema: si la acción que supone el intelecto agente no es propia de la mente particular sino algo común a todas ellas, entonces no se daría una pluralidad en sentido estricto. ${ }^{11} \mathrm{La}$ estrategia de Blank para enfrentar este problema es mostrar que a lo largo de la historia se encuentran concepciones en las que se sostiene un único intelecto agente al mismo tiempo que se reconoce una pluralidad de sustancias. De esta manera, Blank aspira a mostrar que Leibniz podría haber afirmado un intelecto agente universal sin por ello renunciar a un pluralismo de sustancias tipo mentes. Una de tales concepciones que se han dado en la historia, un siglo antes que Leibniz, es la de Alessandro Achillini (1463-1512), quien de acuerdo con Blank

\footnotetext{
8 Laerke (2008), pp.500-517.

${ }^{9}$ Laerke (20080 pp.488-489.

${ }^{10}$ Blank (2001); Blank (2014). Este exégeta entiende que Leibniz habría distinguido dos sentidos del término 'sustancia', a saber, por una parte sustancia como lo que no requiere de otra cosa para existir, y por otra parte como una cosa activa. En consecuencia, de acuerdo con Blank para Leibniz hay un monismo sustancial (Dios como la sustancia que no requiere de otra cosa para existir) al mismo tiempo que un pluralismo de sustancias tipo mentes (como cosas activas). Hemos abordado la cuestión del monismo y el pluralismo en los escritos de DSR, en relación con el problema del continuo, en Raffo Quintana (2013). 11 Blank (2014).
} 


\section{Dossiê Leibniz, Dissertatio - Volume Suplementar 03 | UFPel [2016]}

“(...) defiende que la teoría de un intelecto agente universal es compatible con la visión de que las mentes humanas son genuinas sustancias poseedoras de acciones cognitivas espontáneas". ${ }^{12}$ En pocas palabras, para Achillini hay un único intelecto agente universal que se identifica con el primer motor, un único intelecto paciente capaz de entender las formas abstraídas por el intelecto agente, así como también cada cuerpo humano está dotado de una forma sustancial propia que no es el intelecto agente sino aquello que le confiere a cada ser humano la capacidad racional, de modo que, aunque el intelecto agente sea uno, lo mismo que el paciente, hay una pluralidad de formas sustanciales y, con ello, una pluralidad de sustancias que lo poseen. ${ }^{13}$ Como se dijo, no es la intención de Blank afirmar que Leibniz conoció el pensamiento de Achillini, sino simplemente mostrar que existieron históricamente interpretaciones no monistas del intelecto agente universal.

Ahora bien, las dos líneas interpretativas mencionadas anteriormente, esto es, la que sostiene un monismo sustancial y la que mantiene además un pluralismo de sustancias activas en DSR, han entendido que Leibniz afirmó una concepción averroísta del intelecto agente. No obstante, como veremos, Leibniz nunca afirmó esto en el contexto de DSR. En este trabajo mostraremos que la afirmación de Leibniz de que "en la mente hay algo divino, lo que Aristóteles llamaba intelecto agente", admite una interpretación distinta, que se desprende de los textos de Leibniz y que no es afín con una expresión de tipo averroísta.

12 Blank (2014), p.234.

${ }^{13}$ Blank (2014), p.235-241. 


\section{Federico Raffo Quintana}

\section{El pluralismo de entes}

Para cumplir con nuestro objetivo es necesario que, en primer lugar, examinemos la tesis pluralista, pues desde aquí se desprenderá que, cuando Leibniz se refiere al 'intelecto agente', no piensa en un intelecto universal. La afirmación de una pluralidad de sustancias se enfrenta con una gran dificultad, a saber, que en los escritos de DSR Leibniz utiliza pocas veces la noción de sustancia y lo hace para referirse a Dios como sujeto de todos los atributos afirmativos compatibles. ${ }^{14}$ En este sentido, no encontramos un texto en el que Leibniz diga explícitamente que hay un pluralismo de sustancias. Sin embargo, Leibniz reserva una denominación especial para referirse a las mentes, tanto la divina como las restantes, a saber: ellas son entes. Leibniz emplea el concepto de ente, por una parte, para diferenciar a Dios de las cosas: “(...) solemos decir que 'Dios es un Ente', pero no solemos decir que 'Dios es una cosa". ${ }^{15}$ Sin embargo, por otra parte, el concepto de ente no es exclusivo de Dios sino de las mentes en general, pues para Leibniz las mentes son "los verdaderos Entes" (omnia vera Entia seu Mentes). ${ }^{16}$ Esta tesis de Leibniz implica un cambio importante en relación con sus planteos previos. Por ejemplo, en una célebre carta que Leibniz le envió a J. Thomasius en abril de 1669, afirmó que no se da en el mundo

\footnotetext{
${ }^{14}$ Por ejemplo, De formis seu attributis Dei, AA VI 3, p.514 y Quod Ens perfectissimum sit possibile, A VI 3573.

${ }^{15}$ De origine rerum ex formis, AA VI 3, p.519. "(...) solemus dicere Deus est Ens, non solemus dicere Deus est res".

${ }^{16}$ De veritatibus, de mente, de Deo, de universo, AA VI 3, p.510. Leibniz menciona esto en relación con el hecho de que las mentes crecen en perfección. Como veremos en lo siguiente, para Leibniz esto significó que ellas crecen en el conocimiento.
} 


\title{
Dossiê Leibniz, Dissertatio - Volume Suplementar 03 | UFPel [2016]
}

ningún ente exceptuando la mente, el espacio, la materia y el movimiento. ${ }^{17}$ No es nuestra intención aquí centrarnos en todas las consecuencias que podría tener este cambio en el pensamiento de Leibniz. Basta con señalar que en el contexto de DSR, Leibniz sostuvo un pluralismo de 'entes' tipo mentes.

Ahora bien, el hecho de que pueda hablarse de una pluralidad de entes se funda, al menos parcialmente, en el hecho de que puede reconocerse que hay algo que permanece idéntico en cada mente. Para Leibniz se trata, en cada caso, de algo particular. Así, por ejemplo, señala:

\begin{abstract}
En nuestra Mente hay una percepción o sensación de sí mismo, en tanto que una cierta cosa particular que está siempre en nosotros, puesto que siempre que aplicamos una palabra, entonces reconocemos al instante [que hemos hecho] esto. (...). Esto es lo que vulgarmente llamamos 'idéntico', esta facultad en nosotros independiente de las cosas externas. ${ }^{18}$
\end{abstract}

Leibniz se refiere también a "la identidad de la mente" (mentis identitas) o a la "memoria intelectual" (memoria intellectualis) para hablar de lo 'idéntico' en cada mente. ${ }^{19}$ De alguna manera, pareciera que, para ser un 'ente', no es necesario ser 'sustancia', aunque sí poseer una cierta identidad mental. Ahora bien, Leibniz nota que hay algo que permanece en una mente, “(...) como algo particular que posee ciertas modificaciones, lo que, a saber, percibe estas o

\footnotetext{
${ }^{17}$ Leibniz a Thomasius, AA II 1, p.34.

${ }^{18}$ De veritatibus, de mente, de Deo, de universo, AA VI 3, p.509. "Mente nostra est perceptio seu sensus sui, ut certae cuiusdam rei particularis, haec semper in nobis, quia quoties vocabulum adhibemus, tunc id statim agnoscimus. (...) hoc est quod vulgo appellamus idem, haec in nobis facultas independens ab externis".

${ }^{19}$ De veritatibus, de mente, de Deo, de universo, AA VI 3, p.509.
} 
aquellas cosas" ${ }^{20} \mathrm{El}$ hecho de que una mente sea concebida por Leibniz como algo que posee modificaciones podría llevar a pensar que la considera como una sustancia. Leibniz conocía la definición de 'modo' que Spinoza presentó en la célebre carta sobre el infinito enviada a L. Meyer en 1663, como afección de la sustancia. ${ }^{21}$ Más aún, cuando Leibniz lee en febrero de 1676 esta carta de Spinoza, hace la siguiente anotación: “[a]sí pues, ¿la definición de un modo no puede ser de alguna manera la definición de una sustancia?". ${ }^{22}$ Si esta pregunta de Leibniz se considera conjuntamente con otra anotación que hizo en el mismo escrito de Spinoza, a saber, que aún no ha sido probado que la sustancia no puede concebirse sino como infinita, ${ }^{23}$ se obtiene un fuerte indicio de que concibió a las mentes como sustancias. Al margen de estas especulaciones, la afirmación de Leibniz de la persistencia de 'lo idéntico’ en la mente singular está estrictamente vinculada con la negación de un intelecto universal. De acuerdo con Leibniz, la identidad de la mente no puede ser destruida por las modificaciones, de tal manera que ella no puede extinguirse o dejar de ser. Sin embargo, para Leibniz las concepciones que sostienen que una mente puede extinguirse y retornar a Dios, afirman en consecuencia un intelecto universal:

\footnotetext{
${ }^{20}$ De veritatibus, de mente, de Deo, de universo, AA VI 3, p.509. "Non video quomodo homo seu mens mori seu extingui possit durantibus illis reflexionibus. Manet aliquid in modificationibus non ut ipsi per se extensio in spatio, sed ut quiddam particulare, ceitis modificationibus praeditum, quod scilicet haec vel illa percepit".

${ }^{21}$ Communicata ex literis domini Schulleri, AA VI 3, p.277.

${ }^{22}$ Communicata ex literis domini Schulleri, AA VI 3, p.277. "Ergone certa ratione definitio modi definitio substantiae esse potest?".

${ }^{23}$ Communicata ex literis domini Schulleri, AA VI, p.3 278.
} 


\section{Dossiê Leibniz, Dissertatio - Volume Suplementar 03 | UFPel [2016]}

Si una Mente ha podido comenzar, podrá extinguirse. Y del mismo modo que ha sido hecha por Dios, también volverá a Dios. Esta será una opinión acorde con la Aristotélica y con aquellas que [hablan] de un intelecto universal. Por el contrario, a mí me parece que nunca un alma ha comenzado o puede cesar. No por esto, sin embargo, una Mente será menos creada por Dios, puesto que existirá y subsistirá por la voluntad de Dios, esto es, de un intelecto bueno. ${ }^{24}$

En consecuencia, la negación por parte de Leibniz del hecho de que las mentes se extingan, conlleva oponerse a la afirmación de una inteligencia universal. Esto explica también que Leibniz se aleje de algunas opiniones de Spinoza que conocía gracias a Tschirnhaus, tales como que lo que constituye el ser actual del alma es la idea de una cosa singular ${ }^{25}$ o que el alma humana es una parte de Dios: ${ }^{26}$

Y así no apruebo la opinión de Spinoza, que la Mente singular se extingue con el cuerpo, que la mente de ningún modo habrá de recordar lo precedente, que reste solamente aquello que es eterno en la Mente, la Idea o esencia del cuerpo, a saber, de este [cuerpo]. ${ }^{27}$

De todo esto resulta que el modo como ha de entenderse la afirmación leibniziana de que el intelecto agente es 'lo divino' en las mentes, no ha de ser pensado en términos de un intelecto universal. En el siguiente apartado

\footnotetext{
${ }^{24}$ De veritatibus, de mente, de Deo, de universo, AA VI 3, p.512. "Si Mens incipere potuit, potent extingui. Et quemadmodum facta est ex Deo, redibit in Deum. Haec foret sententia Aristotelicae consentiens, et iis qui de intellectu universali. Mihi contra videtur, nullam unquam animam cepisse, aut desinere posse. Non ideo tamen minus Mens creabitur a Deo, quia erit et, subsistet Dei voluntate, Id est intellectus boni". 25 Über Spinozas Ethik, AA VI 3, p.385; Ethica II, Pr. 11.

26 Über Spinozas Ethik, AA VI 3, p.384; Ethica. II, Pr. 11, Cor.

${ }^{27}$ De veritatibus, de mente, de Deo, de universo, AA VI 3, p.510. "Itaque Spinosae sententiam non probo, quod Mens singularis cum corpore extinguatur, quod Mens ullo modo meminerit praecedentium, quod supersit tantum id quod in Mente aeternum est, corporis Idea vel essentia, huius scilicet".
} 
propondremos una interpretación alternativa que se sigue de los escritos de Leibniz.

\section{Las mentes y la omnisciencia divina}

En Leibniz lecteur de Spinoza, M. Laerke propone una clave interpretativa de los textos de DSR, a saber, que Leibniz se valdría de términos tradicionales para reformular algunas ideas de Spinoza, puesto que declararse abiertamente a favor de las tesis del filósofo holandés en el siglo XVII era problemático debido a sus resonancias materialistas y naturalistas. En este sentido, por ejemplo, Leibniz se habría referido a la extensión absoluta como 'inmensidad' e incluso 'omnipresencia'. ${ }^{28}$ No obstante, las conclusiones extraídas en el apartado anterior nos permiten sugerir que, al menos en lo que respecta a la afirmación del intelecto agente, sucede más bien lo contrario, esto es, que Leibniz expresa ideas tradicionales en términos de su época, presumiblemente para adaptarse a sus interlocutores (en este caso, por ejemplo, Tschirnhaus). Para aclarar lo que queremos decir con esto, recordemos el pasaje que introdujo la polémica que guía nuestro trabajo: "así como en el espacio hay algo divino, la inmensidad misma de Dios, del mismo modo en la mente hay algo divino, lo que Aristóteles llamaba intelecto agente; y esto es lo mismo que la omnisciencia de Dios". ${ }^{29}$ Aunque la mayoría de los especialistas en el pensamiento de Leibniz se centraron

${ }^{29}$ Notizen zur Wissenchaft und Metaphysik, AA VI 3, p.391. Véase la cita en el original en la nota 5. 


\section{Dossiê Leibniz, Dissertatio - Volume Suplementar 03 U UFPel [2016]}

en la mención del 'intelecto agente', lo cierto es que en DSR Leibniz no utiliza esta expresión más que en esta cita. No obstante, la noción de 'omnisciencia' fue tratada con cierta profundidad. Con el objetivo de mostrar que Leibniz no mantuvo un intelecto agente universal en términos averroístas, a continuación examinaremos la analogía entre inmensidad y omnisciencia.

El punto de partida del examen leibniziano de la extensión por sí o inmensidad está relacionado con la noción cartesiana de 'extensión'. Entre mediados de 1675 y comienzos del año siguiente, Leibniz leyó los Principia Philosophiae de Descartes y dejó múltiples anotaciones y comentarios, muchos de los cuales evidencian un desacuerdo de Leibniz en relación con algunas ideas del filósofo francés. ${ }^{30}$ Por un lado, Leibniz nota que la extensión no puede ser la única nota definitoria del cuerpo, puesto que la impenetrabilidad, que también es una nota esencial del cuerpo, no puede demostrarse a partir de la de largo, ancho y profundidad. ${ }^{31}$ Ahora bien, por otro lado, la diferencia entre los cuerpos y la extensión adquiere una dimensión más profunda en los escritos de DSR, que trasciende la mera cuestión de si la noción de espacio basta o no para definir al cuerpo. En este sentido, la distinción entre 'extensión' y 'cosas extensas' significa que el atributo divino de la extensión y los lugares o espacios ocupados por los cuerpos no son lo mismo:

Como en la duración, así también en la Extensión hay cierta forma simple inteligible por sí, cuya idea está presente en la mente, y que por ello es

\footnotetext{
30 Si bien las referencias a los Principia Philosophiae de Descartes se dan a lo largo de muchos escritos de 1675-1676, una muestra clara de los comentarios de Leibniz puede hallarse en Zu Descartes'Principia Philosophiae, AA VI 3, p.213-217.

${ }^{31}$ Catena mirabilium demonstrationum de Summa rerum, AA VI 3, p.585.
} 


\section{Federico Raffo Quintana}

irresoluble. Por lo tanto, [esta forma simple es] aquello según lo cual las cosas se dicen extensas, aquello a lo cual compete por sí la sola extensión (...). Mientras tanto, será suficiente notar que esta Inmensidad responde a la eternidad, y así como la eternidad no implica por sí una sucesión, así tampoco la inmensidad implica extensión o partes. ${ }^{32}$

Como puede observarse en la cita, cuando Leibniz examina el atributo divino de la extensión o la inmensidad, lo concibe como una forma inteligible por sí que es aquello según lo cual las cosas se dicen extensas. Es posible que Spinoza haya influido en Leibniz en esta cuestión indirectamente, a través de sus conversaciones con Tschirnhaus y de algunas cosas que le fueron comunicadas por G. H. Schuller por correspondencia en febrero de 1676. ${ }^{33}$ En efecto, en unas anotaciones de las conversaciones que mantuvo con Tschirnhaus sobre la Ethica de Spinoza (que el filósofo de Leipzig aún no había leído), ${ }^{34}$ Leibniz subrayó que Descartes se equivocó al señalar que la extensión implica divisibilidad. ${ }^{35}$ No obstante, esto no significa que Leibniz se limite a repetir las ideas de Spinoza. De hecho, por ejemplo, Leibniz entiende que la afirmación spinozista de que la

\footnotetext{
${ }^{32}$ De magnitudine, AA VI 3, p.484. "Ut in duratione, ita in Extensione est forma quaedam simplex per se intelligibilis, cuius idea est praesens menti, quaeque adeo est inexplicabilis. Id ergo secundum quod res dicuntur extensae, cui extensio sola per se competit (...) Illud interea notare suffecerit Immensitatem respondere aeternitati, utque aeternitas per se non dicit successionem, ita nec immensitatem dicere extensionem sive partes.

${ }^{33}$ Este es el caso, por ejemplo, de la mencionada anteriormente carta que Spinoza le envió a L. Meyer el 20 de abril de 1663 conocida como 'carta sobre el infinito'. Así la denomina por ejemplo Tschirnhaus en correspondencia con Spinoza. EP LXXX (IV,, p.331). Véase Communicata ex literis domini Schulleri, AA VI 3, p.275-282.

${ }^{34}$ Tschirnhaus le pidió permiso a Spinoza para comunicarle sus escritos a Leibniz, aunque su pedido no fue aceptado bajo pretexto de que sería una imprudencia dárselos a conocer a Leibniz tan prematuramente. Véase EP LXX (IV, p.302-303), para el pedido de Tschirnhaus a Spinoza por medio de Schuller, así como EP LXXII (IV, p.305), para la respuesta de Spinoza.

35 Über Spinozas Ethik, AA VI 3, p.385.
} 


\section{Dossiê Leibniz, Dissertatio - Volume Suplementar 03 U UFPel [2016]}

sustancia extensa no se compone de partes es lo mismo que Thomas White ha querido probar en el prefacio al Demonstratio immortalitate animae rationalis (1664) de Kenelm Digby al señalar que en el continuo no hay partes actuales. ${ }^{36}$ Sea como fuere, para Leibniz la inmensidad o extensión se presenta, en consecuencia, como una cierta naturaleza que da razón de la unidad en la multiplicidad de las cosas extensas. Si se nos permite decirlo en términos de Spinoza, es un atributo que expresa una esencia infinita en su género. ${ }^{37}$ Leibniz prefiere decir que es absoluto como una perfección que contiene todas las cosas de su género. ${ }^{38}$ En este sentido, para Leibniz Dios no constituye parte de las cosas, sino que es su principio o su base. ${ }^{39}$ En la medida en que se trata de un atributo de Dios, es una perfección. Al respecto, Leibniz señala que "[u]na perfección es un atributo absoluto afirmativo; y [la perfección] contiene siempre todas las cosas de su género, pues no hay nada que lo limite". ${ }^{40}$ Es en este sentido que decíamos antes que la extensión por sí es aquello por lo cual todas las cosas extensas se dicen tales. En consecuencia, en analogía, la omnisciencia, como atributo de Dios, podría entenderse como aquello perfecto en el género de las mentes, o más precisamente del pensamiento o de la scientia; es lo absoluto

\footnotetext{
${ }^{36}$ Digby (1664), prefacio de White, s/n. Con respecto a la comparación hecha por Leibniz entre el planteo de White y de Spinoza, Communicata ex literis domini Schulleri, AA VI 3, p.278. Leibniz cuestionó la interpretación de White en Demonstratio Possibilitatis Mysteriorum Eucharistiae, AA VI 1, p.505 y Theoria motus abstracti, AA VI 2, p.264.

${ }^{37}$ Ethica I Pr. 16.

${ }^{38}$ Notizen zur Wissenschaft und Metaphysik, A VI 3, p.392.

${ }^{39}$ De origine rerum ex formis, AA VI 3, p.519.

40 Notizen zur Wissenschaft und Metaphysik, AA VI 3, p.392. "Perfectio est attributum affirmativum absolutum; continetque semper omnia sui generis, cum nihil sit, quod ipsum limitet".
} 
en relación con lo limitado, esto es, con todo pensamiento que no sea omnisciente al modo divino. En este sentido, por ejemplo, Leibniz señala: “[s]i una mente no piensa algo en particular, pero sin embargo piensa, será Dios, es decir, pensará todas las cosas". ${ }^{41}$ El conocimiento de Dios abarca todo, pues Él es omnisciente; sin embargo, cualquier otro conocimiento, esto es, de las mentes particulares, que no conocen distintamente todo y por lo tanto no son perfectamente omniscientes, se dice tal en su relación con lo perfecto en su género, esto es, la omnisciencia divina. Dios conoce distintamente todas las cosas; sin embargo, las mentes particulares conocen sólo confusamente: “[m]e parece que Toda mente es omnisciente confusamente, y que cualquier Mente simultáneamente percibe todo lo que sucede en todo el mundo". ${ }^{42}$

En consecuencia, lo que Leibniz quiere decir al afirmar que hay algo divino en las mentes no es que la acción no les pertenece propia e individualmente sino que, en el ejercicio de su acción, esto es, pensando, las mentes no conocen todas las cosas distintamente, y que este conocimiento parcial y limitado se dice tal en relación con un conocimiento que no encuentra límites y que es, en consecuencia, absoluto, a saber, el conocimiento de aquello que es omnisciente de un modo perfecto. Para Leibniz, en efecto, "nada impide que haya un pensamiento perfecto, es decir, de todas las cosas". ${ }^{43}$ En este

${ }^{41}$ De veritatibus, de mente, de Deo, de universo, AA VI 3, p.512. "Si Mens quaedam nihil cogitet particulare, sed cogitet tamen, est Deus: seu cogitabit omnia".

${ }^{42}$ De plenitudine mundi, AA VI 3, p.524. "Mihi videtur Omnem mentem esse omnisciam, confuse. Et quamlibet Mentem simul percipere quicquid fit in toto mundo".

${ }^{43}$ De formis seu attributis Dei, AA VI 3, p.514. "At cogitationem perfectam seu omnium esse nil impedit". 


\section{Dossiê Leibniz, Dissertatio - Volume Suplementar 03 U UFPel [2016]}

sentido, Leibniz explica que las mentes (esto es, excluyendo la divina) pueden tener un conocimiento claro y distinto solamente de cosas particulares, pero no de todas las cosas: "[p]ues sin duda es verdadero que yo percibo 'alguna cosa' clara y distintamente y que esto es cierto para mí por ahora, pero no tengo ninguna certeza de que haya entendido 'todas las cosas' clara y distintamente". ${ }^{44}$ De esta manera, cuando Leibniz señala que "en la mente hay algo divino, lo que Aristóteles llamaba intelecto agente; y esto es lo mismo que la omnisciencia de Dios", parece estar entendiendo que la omnisciencia perfecta de Dios, esto es, el conocimiento distinto de todo, es lo mismo que el intelecto agente, esto es, interpretado aristotélicamente, aquel en que están en acto todas las formas inteligibles. Asimismo, entender el atributo de la omnisciencia divina de esta manera es más acorde con la visión de Leibniz de que Dios no ha de ser concebido como algo puramente imaginario e incapaz de pensamiento, de voluntad o en general de acción. En consecuencia, que el atributo divino de la omnisciencia sea lo eterno en las mentes acarrea una concepción de Dios como de “(...) cierta Sustancia, Persona, Mente", es decir, una "sustancia inteligente". 45

\footnotetext{
${ }^{44}$ De veritatibus, de mente, de Deo, de universo, AA VI 3, p.508. "Nam verum quidem est me quid clare distincteque percipere. et certum mihi impraesentiarum, sed nullam habeo certitudinem, quod omnia clare distincteque intellexerim".

${ }^{45} \mathrm{De}$ arcanis sublimium vel de summa rerum, AA VI4, pp. 474-475. "Deus non est quiddam Metaphysicum, imaginarium, incapax cogitationis, voluntatis, actionis, qualem nonnulli faciunt, ut idem futurum sit ac si diceres Deum esse naturam, fatum, fortunam, necessitatem, Mundum, sed Deus est Substantia quaedam, Persona, Mens. Possent inscribi Meditationes istae, de arcanis sublimium, vel etiam de Summa Rerum. (...) Ostendendum est Deum esse personam seu substantiam intelligentem. Rigorose demonstrandum est, quod sentit se agere in se ipsum, nihil enim admirabilius quam idem sentire ac pati a se ipso".
} 


\title{
Federico Raffo Quintana
}

\section{Consideraciones finales}

En síntesis, el hecho de que para Leibniz el intelecto agente sea lo divino en la mente no significa que haya un intelecto universal común a todas las mentes. Más bien, hemos visto que para el filósofo de Leipzig es imposible que haya un intelecto único. No obstante, nada impide que haya un intelecto supremo que conozca todas las cosas clara y distintamente, es decir, de un modo perfecto. En consecuencia, aunque Dios no sea la única inteligencia, es sin embargo la inteligencia primera. En este sentido, dice:

\begin{abstract}
Dios es la inteligencia primera en cuanto es omnisciente, es decir, en cuanto ella contiene la forma absoluta afirmativa que se atribuye limitadamente a las otras cosas que se dicen percibir algo. De la misma manera Dios es lo Inmenso mismo en cuanto que se le atribuye una perfección, esto es, la forma afirmativa absoluta que se halla en las cosas cuando se atribuye a ellas estar en algún lado, estar presentes. ${ }^{46}$
\end{abstract}

Hay dos aclaraciones con las que quisiéramos finalizar este trabajo. En primer lugar, resta aún la pregunta por cómo explicaría Leibniz que las mentes, exceptuada la divina, conocen de un modo limitado. Sin profundizar demasiado en el asunto, Leibniz menciona que las mentes están añadidas a la materia, de manera que sin la materia no percibirían del modo en que lo hacen. En este sentido, para Leibniz es debida a la naturaleza de la materia y de la mente que se producen disposiciones en nuestra mente, tales como la de la luz, el

\footnotetext{
${ }^{46}$ De origine rerum ex formis, AA VI 3, p.520. "Deus est intelligentia prima, quatenus est omniscius, seu quatenus eam continet formam absolutam affirmativam, quae limitate tribuitur aliis quae aliquid percipere dicuntur. Quemadmodum Deus est ipsum Immensum, quatenus perfectio ei tribuitur id est forma affirmativa absoluta quae invenitur in rebus, cum tribuitur ipsis esse alicubi, esse praesentes".
} 


\title{
Dossiê Leibniz, Dissertatio - Volume Suplementar 03 | UFPel [2016]
}

enrojecimiento y otras semejantes. ${ }^{47}$ De esta manera, la 'omnisciencia confusa' puede ser explicada como percepción desde un punto de vista. Para explicar esto, el filósofo de Leipzig recurre a la célebre imagen de una ciudad vista desde distintos lados:

\begin{abstract}
Además, no es admirable que una mente perciba las cosas que suceden en todo el mundo, puesto que no hay cuerpo tan exiguo que, supuesta la plenitud del Mundo, no sienta todas las demás cosas. Y así, de este modo, surge una admirable variedad: en efecto, las mentes son tantas como las diversas relaciones del universo, como si se contempla la misma ciudad desde diversos lugares. Y así, con la creación de muchas Mentes Dios quiso producir, respecto del universo, lo que, respecto de una gran ciudad, un pintor que quisiera exhibir varias imágenes o proyecciones pintadas de la misma; el pintor en la tabla como Dios en la mente. ${ }^{48}$
\end{abstract}

En segundo lugar, debemos reconocer que la analogía que Leibniz establece entre la inmensidad respecto del espacio y el intelecto agente respecto de la mente tiene dos limitaciones. Por un lado, hay una limitación en el hecho de que, mientras las mentes son entes, el espacio no lo es. Esta diferencia se observa cuando Leibniz compara el Espacio Universal, esto es, el agregado de todos los lugares ${ }^{49}$ y la República Universal, esto es, la Sociedad de todas las Mentes, pues mientras los lugares o intervalos se destruyen o extinguen, las

\footnotetext{
${ }^{47}$ De origine rerum ex formis, AA VI 3, p.518.

${ }^{48}$ De plenitudine mundi, AA VI 3, p.524. "Porro mirum non est quandam mentem percipere quae aguntur in toto mundo, quia nullum est corpus tam exiguum, quin posita Mundi plenitudine alia omnia sentiat. Itaque hoc modo mira oritur varietas, quot enim mentes, tot diversae universi relationes; quemadmodum si urbs eadem e diversis locis spectetur, itaque plurium Mentium creatione Deus efficere voluit de universo, quod pictor aliquis de magna urbe, qui varias eius species sive projectiones delineatas exhibere vellet, pictor in tabula, ut Deus in mente".

${ }^{49}$ De origine rerum ex formis, AA VI 3, p.519.
} 
mentes no lo hacen. ${ }^{50}$ Por otro lado, y como consecuencia de lo anterior, hay una diferencia en la medida en que las mentes, los 'verdaderos entes', siempre crecen en perfección, ${ }^{51}$ aunque no suceda lo mismo con los cuerpos. A propósito de ellos, Leibniz menciona que el hecho de que no crezcan en perfección es una verdadera razón a priori de por qué las fuerzas siempre permanecen iguales (esto es, de la formulación leibniziana de la ley de conservación). ${ }^{52}$ En contraposición, el perfeccionamiento de las mentes no significa otra cosa que un crecimiento en el conocimiento: "[s]iempre la misma potencia, no siempre la misma ciencia nuestra", ${ }^{53}$

\section{Ediciones de escritos de Leibniz}

LEIBNIZ, G. W. Sämtliche Schriften und Briefe, Darmstadt/Leipzig/Berlin, 1923 yss.

De summa rerum. Metaphysical Papers, 1675-1676.

PARKINSON, G. H. R. (traducción e introducción., New Haven y Londres: Yale University Press, 1992.

50 De origine rerum ex formis, AA VI 3, p.521.

${ }^{51}$ De veritatibus, de mente, de Deo, de universo, AA VI 3, p.510.

52 De origine rerum ex formis, AA VI 3, p.522.

${ }^{53}$ De origine rerum ex formis, AA VI 3, p.522. "Eadem semper potentia, non eadem semper scientia nostra". 
trigonométrie sans tables trigonométriques qui en est le corollaire. PARMENTIER, M. (introducción, traducción y notas) e KNOBLOCH, E. (ed.). París: Vrin, 2004.

\section{REFERENCIAS BIBLIOGRÁFICAS}

AVERrOES. Tafsir del De Anima: Sobre el Intelecto". LORCA, A. M. (introducción, traducción y notas), Éndoxa: Series Filosóficas,17, pp. 9-61. 2003.

BLANK, Andreas. "Substance Monism and Substance Pluralism in Leibniz's Metaphysical Papers 1675-1676”. Studia Leibnitiana, 33, 2, pp.216-223, 2001. . "Leibniz, Spinoza y el intelecto agente". En: CABANAS, L

y ESQUISABEL, O. M. (eds.). Leibniz, frente a Spinoza. Una interpretación panorámica, Granada: Comares, 2014, pp.231-244.

BROWN, Stuart (ed.). The young Leibniz, and his philosophy (1646-76), Dordrech/Boston/London: Kluwer Academic Publishers, 1999.

CABAÑAS, Leticia, y ESQUISABEL, Oscar M. (eds.). Leibnir frente a Spinoza. Una interpretación panorámica, Granada: Comares, 2014.

DE TOMMASO, Emilio M. "From the flos substantiae to the vortex: a shift in Leibniz's early philosophy”. En Pacific Philosophical Quarterly, 95, pp.87-108, 2014.

DESCARTES, René. Oeuvres de Descartes. ADAM, C. y TANNERY, P. (eds.). Paris: Vrin, 1964-1974. 
DIGBY, Kenelm. Demonstratio immortalitatis animae rationalis (prólogo de Thomas White), Frankfurt, 1664.

HOFMANN, Joseph E. Leibniz in Paris, 1672-1676. His growth to mathematical maturity, Cambridge y New York: Cambridge University Press, 1974.

KULSTAD, Mark A. “Leibniz, Spinoza y Tschirnhaus. Metafísica «a tres manos», 1675-1676”. n: CABANAS, L y ESQUISABEL, O. M. (eds.). Leibniz frente a Spinoza. Una interpretación panorámica, Granada: Comares, 2014, pp. 57-76. LAERKE, Mogens. Leibniz lecteur de Spinoza. La genèse d'une opposition complexe. Paris: Honoré Champion Éditeur, 2008.

MERCER, Christia. Leibniz's Metaphysics. Its origins and development. Cambridge: Cambridge University Press, 2004.

RAFFO QUINTANA, Federico. "De summa rerum: monismo y pluralismo en la concepción leibniziana del continuo". Éndoxa: Series Filosóficas, 31, pp.31-45, 2013.

SPINOZA, Baruch. Opera: Im Auftrag der Heidelberger Akademie der Wissenschaften GEBHARDT, C. (ed.). Heidelberg: Carl Winters Universitätsbuchhandlung, 4 vols., 1924. (Se seguirá la manera estándar de citar: Ethica para la Ethica More Geometrico Demonstrata, seguido del número de parte, indicación de proposición, definición, escolio, etc. y número; para la correspondencia, EP, seguido del número de carta en números romanos y luego del tomo y página en números arábigos). 
WILSON, Catherine. "Atoms, Minds and Vortices in De summa rerum: Leibniz vis-a-vis Hobbes and Spinoza". En: BROWN, Stuart (ed.). The young Leibniz and his philosophy (1646-76), Dordrech/Boston/London: Kluwer Academic Publishers: 224-243, 1999. 